\title{
Design and Implementation of a Massively Parallel Version of DIRECT
}

JIAN HE

Department of Computer Science, Virginia Polytechnic Institute and State University, Blacksburg, VA 24061, USA

ALEX VERSTAK

Department of Computer Science, Virginia Polytechnic Institute and State University, Blacksburg, VA 24061, USA

LAYNE T. WATSON

Departments of Computer Science and Mathematics, Virginia Polytechnic Institute and State University, Blacksburg, VA 24061, USA

MASHA SOSONKINA

Ames Laboratory, Iowa State University, Ames, IA 50011, USA

\begin{abstract}
This paper describes several massively parallel implementations for a global search algorithm DIRECT. Two parallel schemes take different approaches to address DIRECT's design challenges imposed by memory requirements and data dependency. Three design aspects in topology, data structures, and task allocation are compared in detail. The goal is to analytically investigate the strengths and weaknesses of these parallel schemes, identify several key sources of inefficiency, and experimentally evaluate a number of improvements in the latest parallel DIRECT implementation. The performance studies demonstrate improved data structure efficiency and load balancing on a 2200 processor cluster.
\end{abstract}

Keywords: data structures, DIRECT, global search, load balancing, task allocation

\section{Introduction}

The availability of compute power on large scale parallel systems offers both potential and challenges for solving high dimensional scientific optimization problems using global search algorithms. Many of these algorithms have large memory requirements and strong data dependency that degrade the program scalability and parallel efficiency as more and more processors join the workforce. The global search algorithm DIRECT (Dividing RECTangle) by Jones et al. (1993) [13] is one such algorithm. Several research projects ([19], [10], and [11]) address its parallel design issues on large systems. Baker et al. ([19]) discuss the performance of several load balancing strategies for a fully distributed version of DIRECT, which solved a 28-dimensional problem on a 256 processor supercomputer. He et al. in [10] and [11] tested two different parallel schemes with various problem scales on a 200 node Opteron cluster of workstations. The intent here is to present the history of these evolving parallel DIRECT implementations. Finally, the current improved parallel scheme is

explored analytically and experimentally on System X, a 2200 processor Apple Xserve G5 cluster 


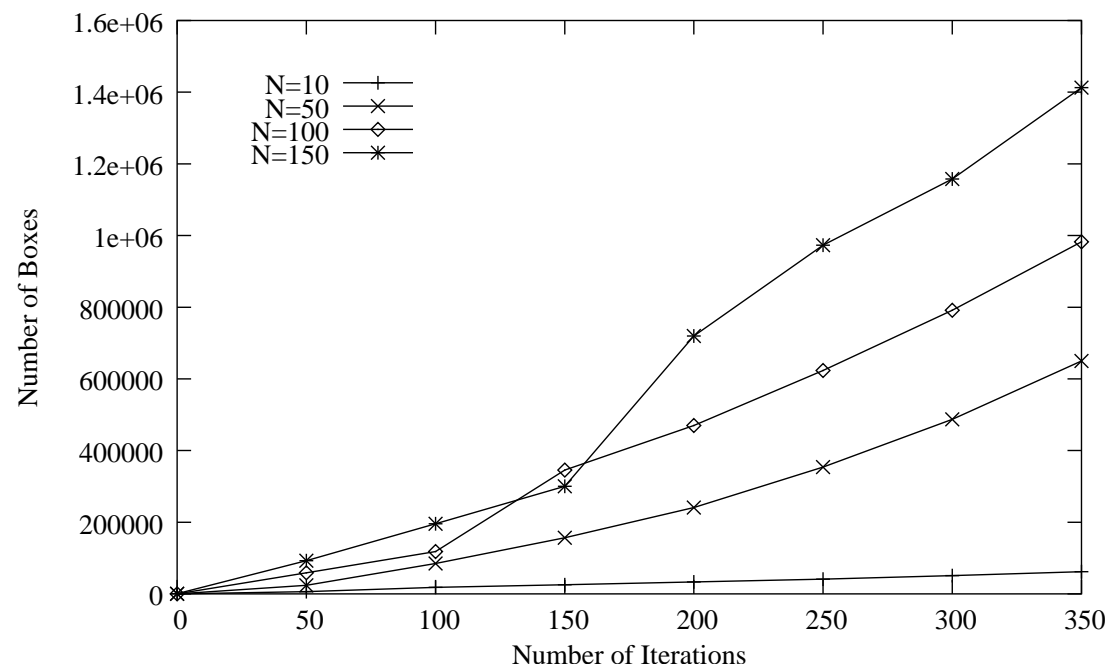

Figure 1.1. The growth of number of boxes for a test problem with dimensions $N=10,50,100$, and 150 .

at Virginia Polytechnic Institute and State University. The performance studies focus on data structure efficiency and load balancing.

In the past decade, DIRECT has been successfully applied to many modern large scale multidisciplinary engineering problems ([2], [3], and [9]). Recently, DIRECT has been used in global nonlinear parameter estimation problems in systems biology [15]. However, unnecessary overhead and complexity caused by inefficient implementation inside other software packages (e.g., Matlab) may obscure DIRECT's advanced features. Some computational biologists are attracted by its unique strategy of balancing global and local search, its selection rules for potentially optimal regions according to a Lipschitz condition, and its easy-to-use black box interface. Like other global optimization approaches of [4] and [6], DIRECT is being challenged by high-dimensional $(\geq 50)$ problems including nonlinear models for parameter estimation. The present work applies DIRECT to a 143-parameter estimation problem for a budding yeast cell cycle model [17].

As the scale of both computational problems and clusters of workstations has grown, parallel optimization algorithms have become a very active research area. However, the nature of the DIRECT algorithm presents both potential benefits and difficulties for a sophisticated and efficient parallel implementation. Gablonsky [5] and Baker et al. [19] are among the few parallel DIRECT implementations known in the public domain. In [5], Gablonsky adopts a master-slave paradigm to parallelize the function evaluations, but little discussion is given to the issue of parallel performance and potential problems, such as load balancing and interprocessor communication, both of which raise many challenging design issues. A major contribution in [19] is a distributed control version equipped with dynamic load balancing strategies. Nevertheless, that work did not fully address other design issues such as a single starting point and a strong data dependency.

At a high level, DIRECT performs two tasks - maintaining data structures that drive its selection of regions to explore and evaluating the objective function at chosen points. One of the limitations of DIRECT lies in the fast growth of its intermediate data. Jones ([14]) comments that DIRECT suffers from the curse of dimensionality that limits it to low dimensional problems $(<20)$. Figure 1.1 shows the growth of the number of search subregions ("boxes") for a standard test problem with dimensions $N=10,50,100$, and 150. The amount of data grows rapidly as the DIRECT search proceeds in iterations, especially for high dimensional problems. This dictates techniques to reduce the size of data structures, thus the number of machines required to hold the distributed data in memory. The second task of evaluating the objective function at sample 


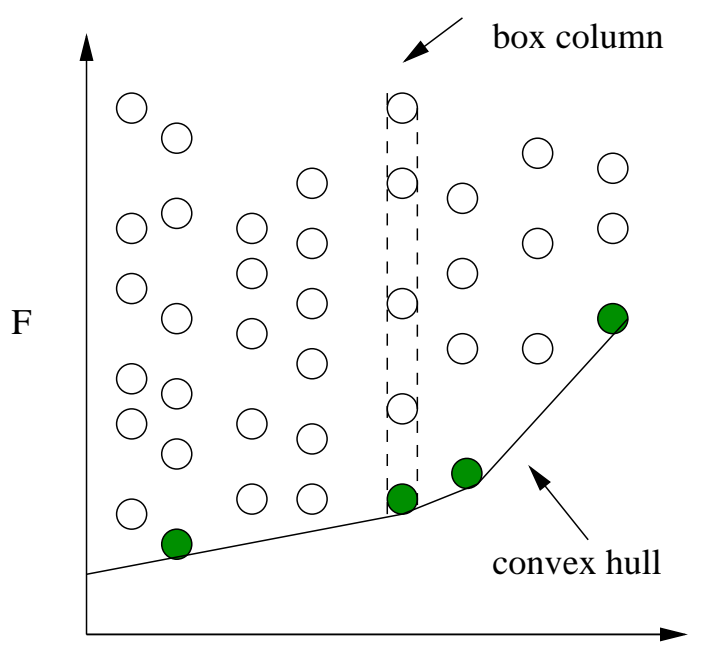

$\mathrm{D}$

Figure 2.1. An example scatter plot of boxes. The $F$-axis is function values, and the $D$-axis is box diameters.

points presents its own challenges. The selection of sample points in the current iteration depends on all the points that have been sampled previously. Empirically, the inherent inefficiency of this necessary point sampling has a great impact on load balancing. Several strategies proposed in the parallel implementation of DIRECT are explored analytically and experimentally.

The paper is organized as follows. Section 2 begins with an overview of the DIRECT algorithm and its parallel design challenges. Section 3 presents two parallel schemes of DIRECT and discusses the evolution of the parallelization process. Performance studies for the latest parallel DIRECT version are presented in Section 4.

\section{Algorithm overview and design challenges}

DIRECT finds the global minimum of an objective function $f(x)$ inside an $N$-dimensional design space $\ell \leq x \leq u$. Each iteration of DIRECT consists of the following three main steps.

1. SELECTION identifies a set $S$ of "potentially optimal" boxes that are subregions inside the design domain with dimension $N$. A box is potentially optimal if, for some Lipschitz constant, the objective function value at its center is potentially smaller than that in any other box (a formal definition of potential optimality can be found in [13]).

2. SAMPLING evaluates new points sampled around the centers of all "potentially optimal" boxes in $S$ along their longest dimensions.

3. DIVISION subdivides "potentially optimal" boxes in $S$ based on the function values at the newly sampled points.

These three steps repeat until the stopping condition is satisfied. Initially, only one box exists in the system. As the search progresses, more boxes are generated, illustrated by the scatter plot shown in Figure 2.1 in which each circle represents a box. The sizes of boxes increase along the $D$ axis (diameter) and the function values at box centers increase along the $F$-axis (function). All the boxes with the same diameter belong to a "box column". Reference [13] proves that all potentially optimal boxes in $S$ are on the lower right convex hull of the scatter plot points in Figure 2.1. Here, 
Functional Components

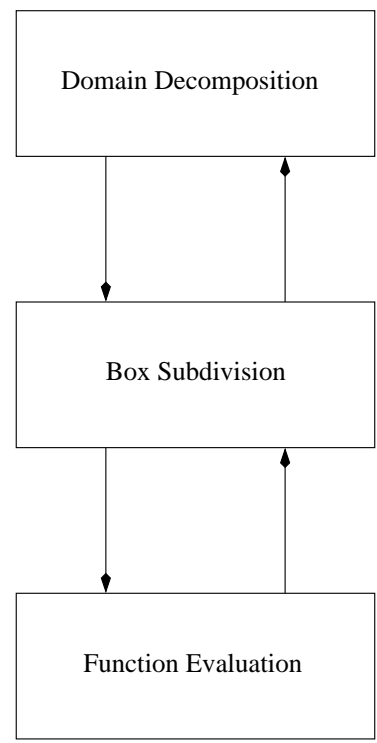

Figure 3.1. Three functional components.

$\epsilon=0$ in the definition of "potentially optimal" in [13]. Call these boxes "convex hull boxes" and the boxes with the lowest function values in each box column "lowest boxes".

In the DIVISION step, multiple new boxes are generated for each convex hull box. The multiple function evaluation tasks at each iteration give rise to a natural functional parallelism used in [5] and [19]. This is especially beneficial for expensive objective functions, since the communication cost of distributing evaluation tasks to multiple processors is negligible compared to the computational cost. On the other hand, a few design challenges are also observed here. First, the algorithm starts with one box, which produces simply one evaluation task for all the acquired processors. With a single starting point, load balancing is always an issue at an early stage, even though the situation will be improved as the search progresses by subdividing the domain and generating multiple evaluation tasks. When a large number of processors is used, the load balancing issue is more critical for low dimensional problems $(<20)$, which subdivide fewer boxes at every iteration than high dimensional problems. For iterations that generate fewer new boxes, a load imbalance occurs with some processors sitting idle. Second, the number of boxes subdivided at each iteration is unpredictable depending on the result of identifying convex hull boxes. For high dimensional problems, the number of boxes grows more rapidly (as shown in Figure 1.1), challenging data structure expandability and memory capacity. Therefore, data parallelism is considered here as a remedy, whereby data is distributed across multiple machines. Third, a strong data dependency that exists throughout the algorithm steps lessens program concurrency, thus degrading parallel scalability. Efficient task and data distribution strategies are demanded here for a scalable parallel DIRECT implementation, especially for large scale systems that host hundreds and thousands of processors.

\section{Parallel schemes}

All the observations above engendered a combined functional and data parallelization in two parallel implementations of the DIRECT algorithm, called pDIRECT_I and pDIRECT_II in what follows. 
The algorithm operations can be partitioned into three functional components: domain decomposition, box subdivision, and function evaluation as shown in Figure 3.1. Domain decomposition is an optional component that transforms the single start DIRECT into a multistart algorithm. Moreover, the resulting multiple subdomains are optimized independently, so that the objective function value may be reduced faster [10]. The domain decomposition is accomplished in the following two phases.

1. The longest dimension of the $N$-dimensional domain is subdivided into $s=\sqrt{m}$ partitions, where $m$ is the desired number of subdomains.

2. Inside each partition above, the longest dimension is subdivided into $s$ parts.

As the second component, box subdivision applies data parallelism that spreads data across multiple processors collaborating on the SELECTION and DIVISION steps. Lastly, the function evaluation component uses the classical master-slave paradigm that distributes evaluation tasks to multiple processors during SAMPLING.

To store the unpredictable number of boxes, both pDIRECT_I and pDIRECT_II reuse the set of dynamic data structures presented in [12]. In addition, a few techniques are developed for pDIRECT_II to reduce local memory storage and network traffic. To distribute data and computation, pDIRECT_I combines a shared memory model (for box subdivision) with a message passing model (between box subdivision and function evaluation), and dynamically spawns processes for these two components. The data is distributed through the global data structures in the shared memory and computational tasks are distributed via messaging. This mixed paradigm improves data distribution efficiency compared to the pure functional parallel versions in [5] and [19]. However, it has its own shortcomings in program portability, processor utilization, load balancing, and termination efficiency. Therefore, the second version pDIRECT_II was developed to address these inefficiencies with a pure message passing model and more dynamic features in data structures, task allocation, and the termination process. Performance results prove that pDIRECT_II is effective for solving complex design optimization problems on modern large scale parallel systems. The following two sections first present pDIRECT_I and its design drawbacks, and then discuss the considerations leading to the improved version pDIRECT_II.

\section{1. $p D I R E C T \_I$}

The parallel scheme of pDIRECT_I consists of three levels as shown in Figure 3.2, each level addressing one of the functional components in Figure 3.1.

\subsubsection{Topology}

The processes at Level 1 form a logical subdomain master ring. The entire design domain is decomposed into multiple nonoverlapping subdomains. Each process $S M_{i}$ (subdomain master $i$, $i=1, \ldots, m)$ on the ring starts the DIRECT search at the center of a chosen subdomain $i . S M_{i}$ detects the stopping conditions, merges the results, and controls the termination at the end. $S M_{i}$ is spawned at run time by MPI and joins the logical ring formed for $S M$ processes. Level 1 uses a ring topology, because it fits the equal relationship among subdomains and represents the dependency of the stopping condition of each subdomain on other subdomains. The overall termination condition

is when all subdomains have satisfied the specified search stopping criteria. In other words, a subdomain will be kept active until all search activities in other subdomains are done. This rule aims at reducing processor idleness when subdomains generate different amounts of computation. The drawback is that the stopping condition (i.e., maximum number of iterations) becomes a lower 


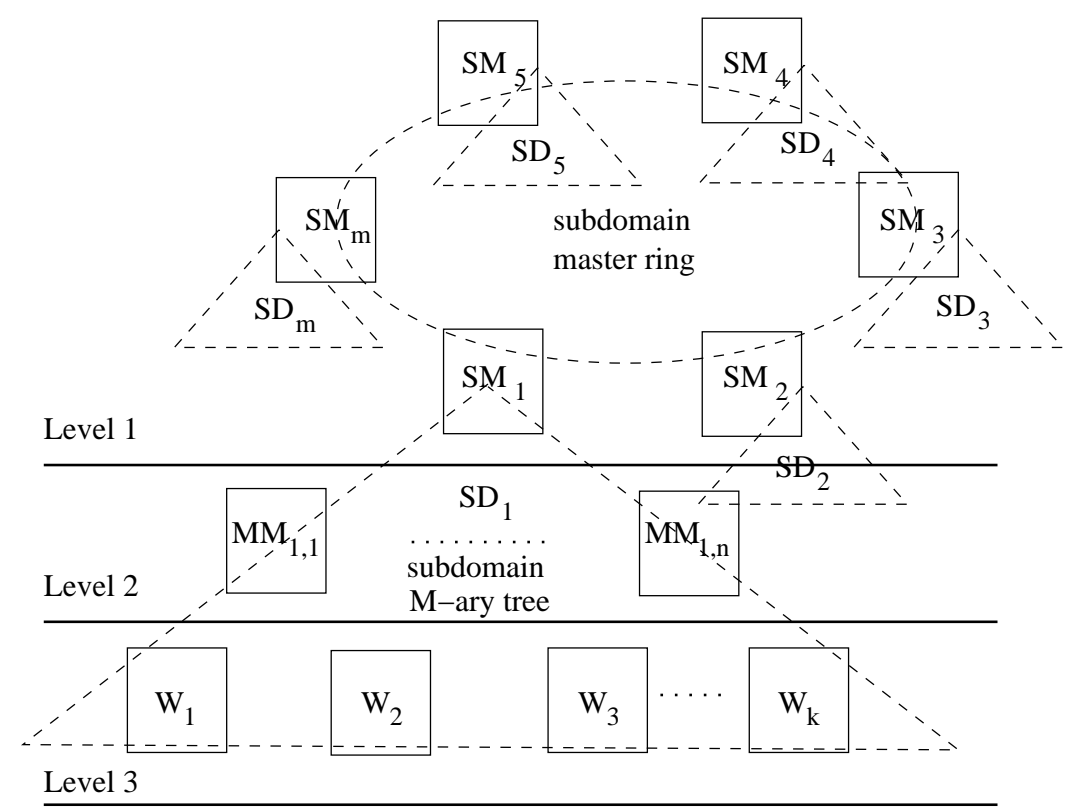

Figure 3.2. The parallel scheme of pDIRECT_I.

bound on the computational cost instead of an exact limit. Furthermore, the termination process is controlled by a token $T$ passed as described in the following.

1. $S M_{1}$ issues $T$ and passes it around the ring.

2. After the local stopping criteria are met, each $S M_{i}$ checks if $T$ has arrived at each iteration. If not, DIRECT proceeds. If yes, $T$ is passed along in the ring.

3. After $T$ is passed back to $S M_{1}$, a termination message is sent to all $S M_{i}$.

4. $S M_{1}$ collects the final results from all $S M_{i}$.

This process decentralizes the termination control, thus avoiding the bottleneck at $S M_{1}$ when the number of subdomains $m$ is large. On the other hand, there are a few disadvantages of using the ring. First, the communication latency on a ring is higher than on some other topologies, such as a star or a tree. Second, the lower bound stopping condition can not provide users accurate estimates of computational cost.

Below Level 1, Level 2 uses GPSHMEM [18] to establish a global addressing space to access the data for SELECTION. This globally shared data structure corresponds to a work pool paradigm [7] that dynamically adjusts box subdivision workload among mini subdomain master $(M M)$ processes at Level 2. Between Levels 2 and 3, a master-slave paradigm is used for distributing function evaluation tasks. Both Levels 2 and 3 take advantage of dynamic process management in MPI-2 [8] so that processors are assigned to these two levels at run time with approximately $(p-m) / m$ processors available for each subdomain (out of $p$ total processors). In Figure 3.2, a $\lfloor M\rfloor$-ary tree structure is rooted at each $S M$ process, where

$$
M=\sqrt{\frac{p-m}{m}} .
$$

Each $S M$ process dynamically spawns $n=\lfloor M\rfloor$ mini subdomain master $(M M)$ processes at Level 2 for box subdivision tasks. Similarly, each $M M$ process spawns $\lfloor k\rfloor$ or $\lceil k\rceil$ worker processes for function evaluation tasks, where

$$
k=\frac{p-m(1+\lfloor M\rfloor)}{m\lfloor M\rfloor} .
$$


To form the $\lfloor M\rfloor$-ary tree of processes, pDIRECT_I requires that the total number of processes $P \geq 16$. If the number of available processors $p \geq 16$, then $P=p$. Otherwise, $P$ is set at 16 , so that multiple processes may run on the same physical processor. Pseudocode 3.1 shows the interactions between $M M_{i, 1}$ and $M M_{i, j}(j=2, \ldots, n)$ in subdomain $i(i=1, \ldots, m)$ managed by $S M_{i}$.

done $:=$ FALSE (the search status)

$M M_{i, 1}$ receives DIRECT parameters (problem size $N$, domain $D$, and stopping conditions $C_{\text {stop }}$ ) from $S M_{i}$ broadcast DIRECT parameters to $M M_{i, j}$

do

if $\left(M M_{i, 1}\right)$ then

if (done $=$ FALSE $)$ then

run one DIRECT iteration and merge intermediate results

if $\left(C_{\text {stop }}\right.$ satisfied $)$ then

done $:=$ TRUE

send done to $S M_{i}$

end if

cycle

else

receive a message from $S M_{i}$

if (not a termination message) then

send a handshaking message to $S M_{i}$

broadcast a message to keep $M M_{i, j}$ working

run one DIRECT iteration and merge intermediate results

cycle

else

broadcast a termination message to $M M_{i, j} \mathrm{~s}$

terminate workers

store the merged results

exit

end if

end if

else

$M M_{i, j}$ receives a message from $M M_{i, 1}$

if (not a termination message) then

run one DIRECT iteration and reduce intermediate results

else

exit

end if

end if

end do

$M M_{i, 1}$ sends the final results to $S M_{i}$

Pseudocode 3.1. 


\section{OPTSET}

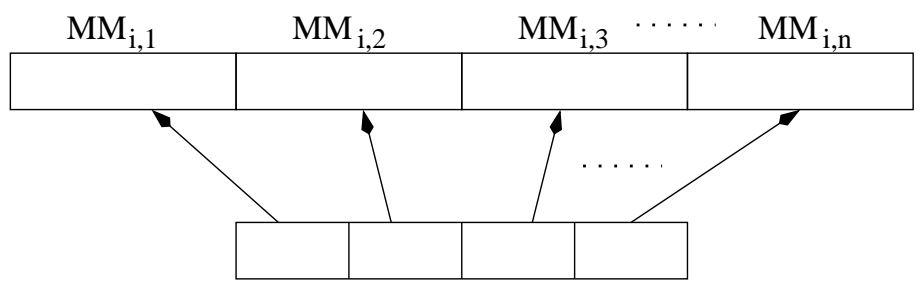

OPTSET INDEX

Figure 3.3. Data structures in GPSHMEM.

The control mechanism is a two-level messaging-between $S M_{i}$ and $M M_{i, 1}$, and between $M M_{i, 1}$ and each $M M_{i, j}$. The DIRECT parameters are passed from $S M_{i}$ to $M M_{i, 1}$, which broadcasts them again to each $M M_{i, j}$. To reduce the control overhead, no handshakes are involved between $S M_{i}$ and $M M_{i, 1}$ before the local stopping criteria are met. However, it is inefficient to dedicate a $S M$ process to monitoring the search status and wrapping up the search at the end, causing significant communication overhead.

\subsubsection{Task allocation}

\subsection{2a. The SELECTION implementation}

At Level 2, $M M$ processes cooperate on identifying convex hull boxes stored in a shared data structure of a global addressing space using GPSHMEM [18]. Two sets of global shared data structures OPTSET and OPTSET_INDEX (Figure 3.3) are used.

The structures OPTSET and OPTSET_INDEX are allocated and distributed across all $M M$ processes, which use one-sided communication operations such as "put" and "get" to access shared data. These one-sided operations provide direct access to remote memory with less interaction between communicating parties. In addition, the data structure LOCALSET is allocated at $M M_{i, 1}$ for merging the boxes with the same size. When only one $M M$ process exists, SELECTION is the same as that in the sequential DIRECT. The following steps describe the SELECTION step implemented in pDIRECT_I.

1. $M M_{i, j}(j=1, \ldots, n)$ puts all the lowest boxes for different box sizes to its own portion in OPTSET and updates its index in OPTSET_INDEX.

2. $M M_{i, 1}$ gets all boxes in OPTSET and merges the boxes with the same size to LOCALSET.

3. $M M_{i, 1}$ finds convex hull boxes in LOCALSET and puts a balanced number of boxes for each $M M_{i, j}$ into OPTSET (the load balancing algorithm at $M M_{i, 1}$ is described in Pseudocode $3.2)$.

4. $M M_{i, j}$ gets its portion of the convex hull boxes from OPTSET, removes some boxes (if any) that are assigned to other $M M_{i, j}$, and starts processing its convex hull boxes.

Each box is tagged with a processor ID and other indexing information to be tracked by its original owner. To minimize the number of local box operations (i.e., removals and additions) and maximize data locality, $M M_{i, 1}$ restores the boxes back to their contributors before it starts load adjustment. The shared memory approach can access more memory on multiple machines than on a single machine. However, it depends on multiple software packages for global addressing, such as GPSHMEM and ARMCI [16]. Secondly, resizing the global data structures to hold an unpredictable number of lowest boxes involves expensive global operations across multiple machines. 
Thirdly, collecting all lowest boxes at $M M_{i, 1}$ burdens local buffer storage as well as network traffic. Lastly, a global barrier is needed between steps to avoid premature "get" operations.

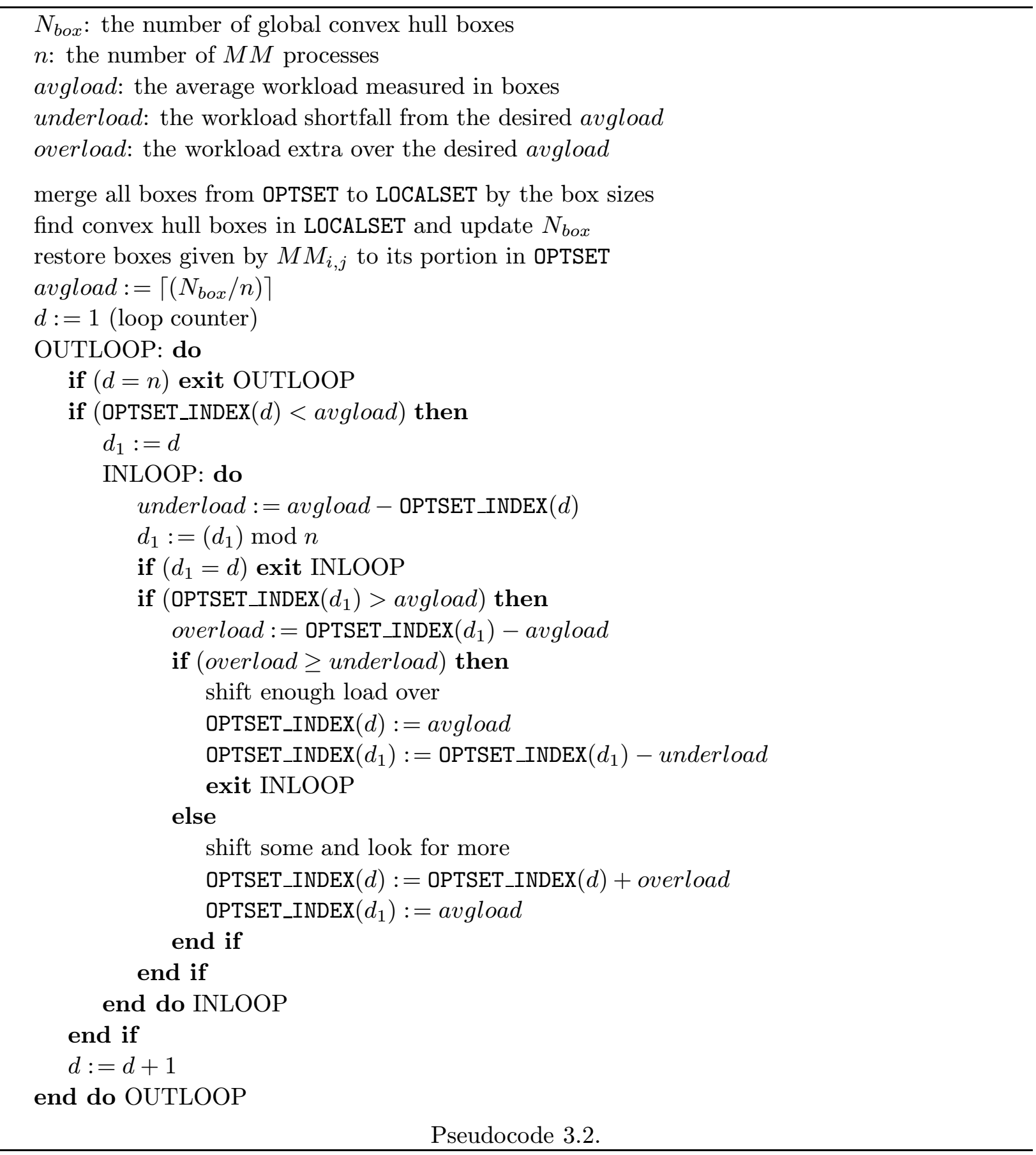

\subsection{2b. Worker assignment}

As shown in Pseudocode 3.2, the load adjustment is done at $M M_{i, 1}$, which distributes the work to $M M_{i, j}$ by using the shared data structures in GPSHMEM. Then, each $M M$ process subdivides its share of convex hull boxes and distributes the function evaluation tasks down to its workers. Although the control mechanism is simple, this centralized strategy suffers a common bottleneck 


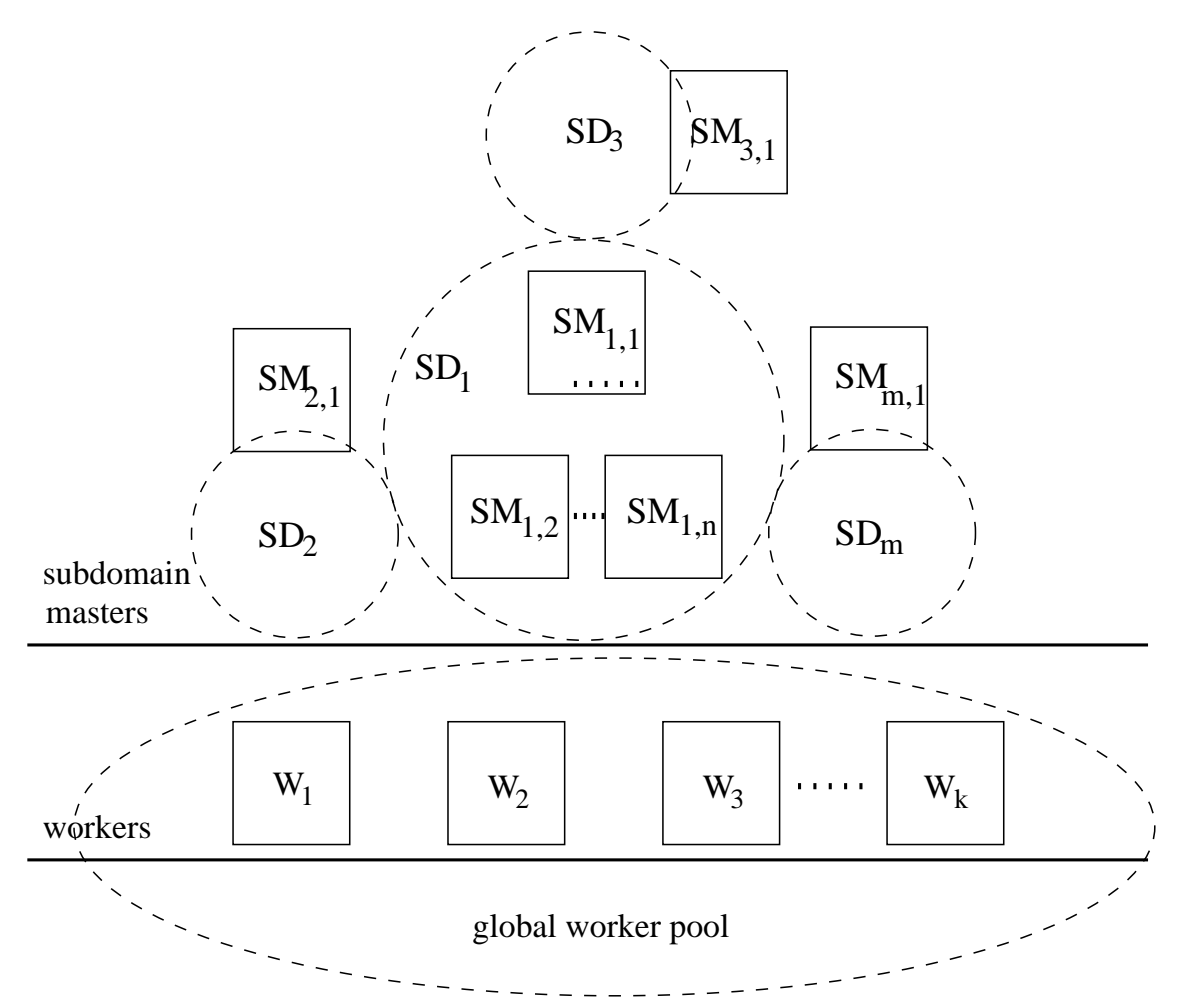

Figure 3.4. The parallel scheme for pDIRECT_II.

problem. Furthermore, workers are not shared by $M M$ processes. A worker is exclusively under the command of a particular $M M$ that spawns it at the beginning. This fixed assignment degrades the processor utilization and load balancing among workers.

\section{2. $p D I R E C T \_I I$}

The three-level hierarchy in pDIRECT_I is reshaped to be a group-pool structure with subdomain groups of masters and a globally shared pool of workers as shown in Figure 3.4. The $S M$ and $M M$ processes in pDIRECT_I are now grouped together to maintain data structures and perform SELECTION and DIVISION, while globally shared workers perform SAMPLING. This scheme is implemented with a pure message passing model, which removes the dependency on multiple software packages, simplifies the program structure, and improves the parallel performance.

\subsubsection{Topology}

Each subdomain is served by a subdomain group of masters in lieu of the subdomain master ring. Let $S M_{i, j}$ stand for the master $j$ in subdomain $i . S M_{i, 1}$ is the root master for subdomain $i$. In addition to carrying out common tasks like other masters, the root masters $S M_{i, 1}(i=2, \ldots, m)$ also communicate with $S M_{1,1}$ to finalize the search. This star shaped connection centered at $S M_{1,1}$ has replaced the ring topology in pDIRECT_I.

Moreover, all $S M_{i, j}(j=1, \ldots, n)$ processes except $S M_{1,1}$ become workers when they have finished all search activities for their subdomain. This dynamic feature reduces the processor idleness and offers an exact stopping condition unavailable in pDIRECT_I. When the stopping condition is satisfied, a termination message is sent from processor 0 down to a logical tree of processors in $\log _{2}(p)$ steps. Recall that the termination message is passed linearly along the ring 
and logarithmically down to the $\lfloor M\rfloor$-ary trees in pDIRECT_I. Clearly, the new termination scheme here does not require such a complicated control mechanism as in pDIRECT_I.

\subsubsection{Data structures}

The data structure design directly affects the efficiency of local memory operations as well as global data distribution. The set of dynamic data structures borrowed from [12] was kept the same in pDIRECT_I, but improved in pDIRECT_II. Limiting box columns (LBC) is a technique developed to reduce the memory requirement.

Let $I_{\max }$ be the maximum number of iterations allowed (a stopping criterion), $I_{\text {current }}$ be the current iteration number, and $C$ be one of the box columns. Each of the iterations $I_{\text {current }}, \ldots, I_{\max }$ can subdivide at most one box from $C$, because at most one box from $C$ can be in the set of convex hull boxes at any iteration. Therefore, $C$ only needs to contain at most $L=I_{\max }-I_{\text {current }}+1$ boxes with the smallest function values. Boxes with larger function values are not considered by the DIRECT search limited to $I_{\max }$ iterations. However, the number of boxes generated per box column is usually much larger than $L$. Figure 3.5 shows the box column lengths for (1) Test Function 6 with dimension $N=10$ and $I_{\max }=400$ and (2) the budding yeast problem with $I_{\max }=40$. (These two functions are defined later in Section 4.) Most of the box columns are longer than $I_{\max }>=L$ in both (1) and (2). When the stopping criterion $I_{\max }$ is given, storing only $L$ boxes in box columns would significantly reduce the memory demands.

Since each box column is implemented as a min-heap ordered by the function values at the box centers, all box column operations without LBC take $\mathcal{O}(\log n)$ time and only two types of heap operations are involved - removing boxes with the smallest function values and adding new boxes. Additionally, LBC needs to remove the boxes with the largest function values $\left(b_{\max } \mathrm{s}\right)$. The min-heap data structure requires a $\mathcal{O}(n)$ time algorithm to locate the $b_{\text {max }}$ boxes. In future work, a min-max heap [1] will replace the min-heap data structure to find the $b_{\text {max }}$ boxes with constant time, which makes all operations $\mathcal{O}(\log n)$ time. The min-max heap makes a huge difference when $I_{\max }$ is large, since the number of boxes in a box column heap is very large. The experimental results in Section 4 show the improvement of pDIRECT_II over pDIRECT_I in reducing local buffer size.

\subsubsection{Task allocation}

Task allocation policies have strong connections to important performance metrics such as parallel efficiency and load balancing. Here, several improvements were made in allocating both box subdivision tasks in the SELECTION step and function evaluation tasks in the SAMPLING step.

\subsection{3a. The SELECTION implementation}

In subdomain $i$, SELECTION is accomplished jointly by masters $S M_{i, j}, j=1, \ldots, n$, where $n$ is the total number of subdomain masters per subdomain. When $n=1$, SELECTION is the same as that in the sequential DIRECT. When $n>1$, SELECTION is done in parallel over the index $i$ as follows.

1. The $S M_{i, j}$ identify local convex hull box sets $S_{i, j}, j=1, \ldots, n$.

2. $S M_{i, 1}$ gathers the $S_{i, j}$ from all the $S M_{i, j}$.

3. $S M_{i, 1}$ merges the $S_{i, j}$ by box diameters and finds the global convex hull box set $S_{i}$.

4. All the $S M_{i, j}$ receive the global set $S_{i}$ and find their portion of the convex hull boxes. 


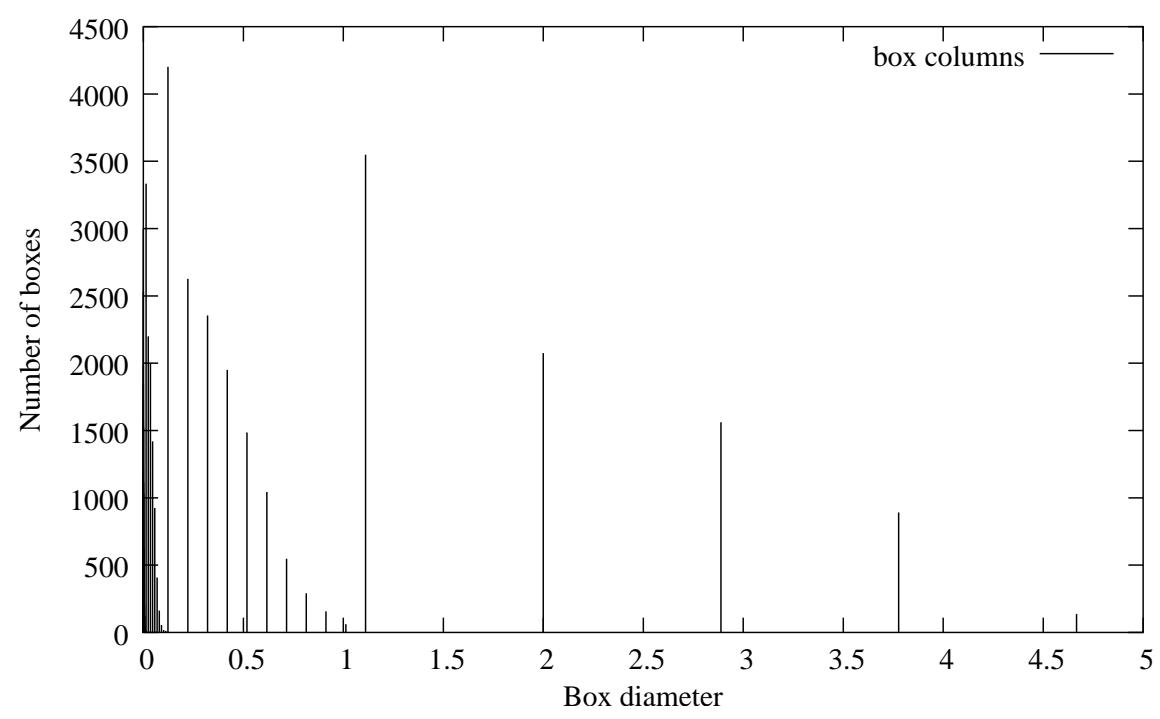

(1) Test Function 6 with $N=10$ and $I_{\max }=400$.

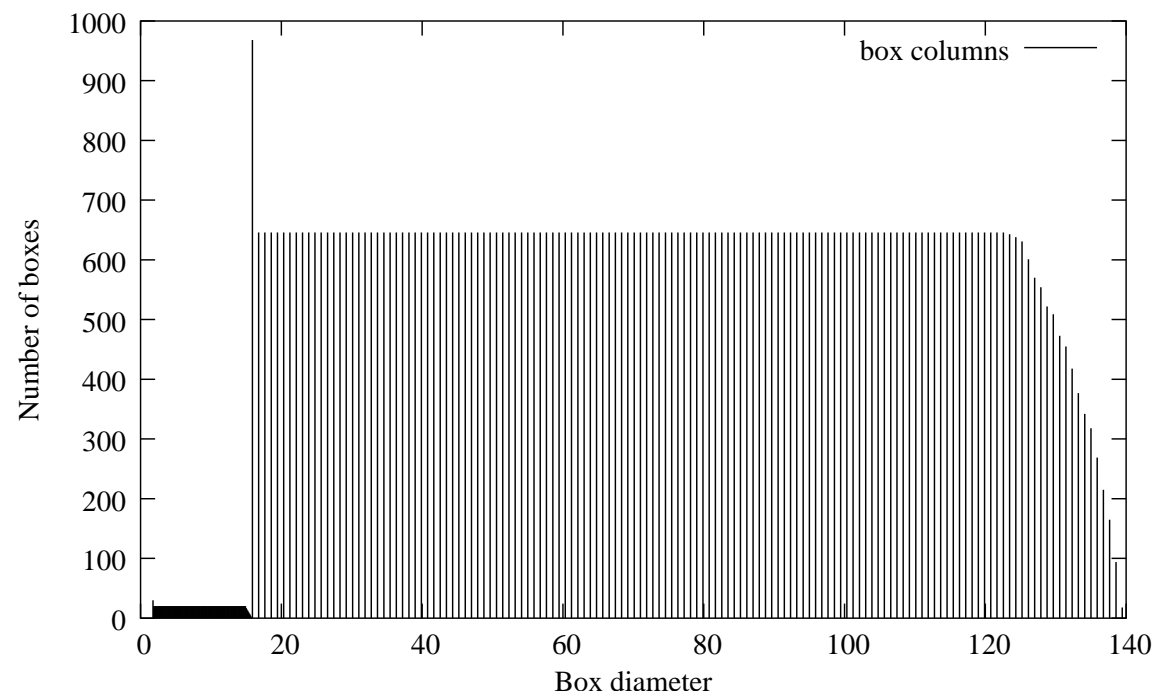

(2) Budding yeast problem, $N=143$ and $I_{\max }=40$.

Figure 3.5. Box column lengths at the last iteration $I_{\max }$.

All buffers used here are locally allocated and resized incrementally according to the updated number of boxes involved in the convex hull computation. Note that all boxes in the global convex hull box set $S_{i}$ must also be in the union of the sets of local convex hull boxes $\left(S_{i, j}\right)$ of the masters. Therefore, each master $S M_{i, j}$ computes $S_{i, j}$ in parallel and $S M_{i, 1}$ only considers the union of all $S_{i, j}$ instead of all the lowest boxes with different diameters, as was done in pDIRECT_I. This decentralized SELECTION implementation reduces memory requirements for buffers, as well as the amount of data transferred over the network. However, a large number of subdomain masters will not perform well due to the global communication and synchronization required for finding convex hull boxes at every iteration. [20] describes a sampling technique that can further reduce the bandwidth requirements, but it comes at the expense of requiring another global communication round. Another possibility would be for $S M_{i, 1}$ to gather via a $d$-way tree only the final merged $S_{i, j}$, where each intermediate tree node does a partial convex hull merge of its $d$ (merged) inputs (the optimal value for $d$ is derived in [20]). 


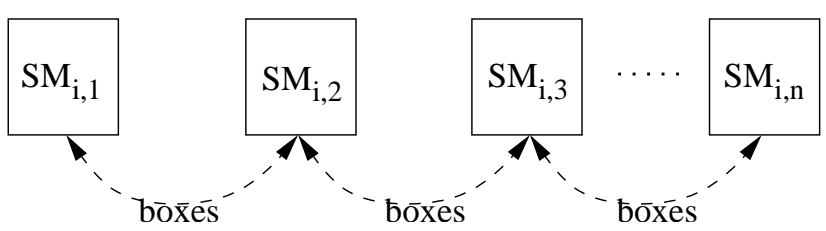

(a) Horizontal 1-D partition.

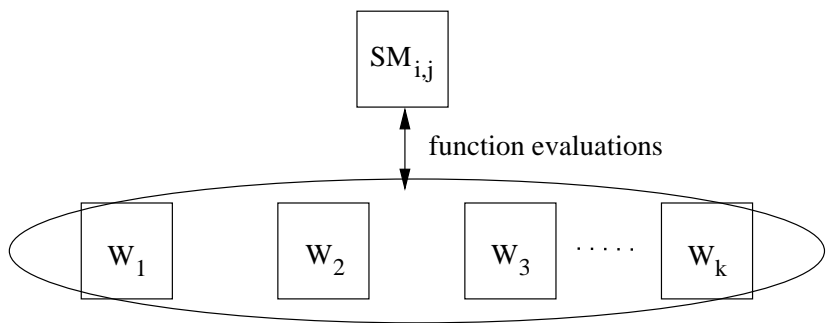

WORKER POOL

(b) Vertical 1-D partition.

Figure 3.6. Task partition schemes.

\subsection{3b. Task partition}

pDIRECT_II supports two ways of task partitioning. In the case of low computation cost, communication overhead dominates the parallel execution time and overshadows the benefits in distributing function evaluation tasks to workers. Wisely, masters would rather keep computation locally and simply share the memory burden with other masters without any worker involved in the picture. This is called horizontal 1-D partition for box subdivision tasks (Figure 3.6(a)). Experimental results in Section 4 show that this scheme achieves better speedup (for cheap function evaluations) than the vertical 1-D partition for function evaluation tasks (shown in Figure 3.6(b)). When these two ways are combined, they become a hybrid 2-D partition. This hybrid scheme is usually preferred for the following reasons. First, the computation cost is higher or at least comparable to the communication cost in most real world design problems. Generally, overlapping the computation on worker processors in the vertical 1-D partition is a reasonable approach. Second, the data sharing scheme in the horizontal 1-D partition relieves the heavy memory burden on a single master processor for solving a large scale and/or high dimensional problem.

\subsection{3c. Worker assignment}

The worker pool is globally shared by all masters in all subdomain groups. Each worker polls all (selected) masters for evaluation tasks and returns the function values. Workers proceed in cycles that roughly correspond to the DIRECT iterations. During each cycle, a worker requests tasks from a randomly selected subset of masters until all of them are out of work. This is called the "nonblocking" loop in Pseudocode 3.3. Once the cycle is over, the worker blocks waiting (the "blocking" loop) for further instructions from a fixed master, which is selected such that every master has a fair number of blocked workers waiting in the queue. Pseudocode 3.3 below describes how a worker $W_{i}$ evaluates the objective function for masters $S M_{i, j}(i=1, \ldots, m$ and $j=1, \ldots$, n) during SAMPLING.

$m$ : the number of subdomains, given as input

loop: the loop status

$C_{\text {active }}$ : the counter for the total number of "active" masters 
that have not reached the last iteration

$C_{i d l e}$ : the counter for idle masters

$P_{w i}$ : the processor ID (PID) of $W_{i}$

mesg: the message received from others

loop $:=$ "nonblocking"

$C_{\text {active }}:=$ current total number of active masters

$C_{\text {idle }}:=C_{\text {active }}-m$ (assume all root subdomain masters busy initially)

OUTLOOP: do

if (loop $=$ "nonblocking") then

send a nonblocking request to a randomly selected master $S M_{i, j}$

from $C_{\text {active }}-C_{\text {idle }}$ busy masters

else

set all masters to status busy

$C_{\text {idle }}:=0$

send a blocking request to the master $S M_{i, j}$ that ranks as

$\left(P_{w i}-C_{\text {active }}\right) \bmod C_{\text {active }}$ in the list of all active masters

end if

INLOOP: do

keep waiting for any messages

select case (mesg)

case (an evaluation task from $S M_{i, j}$ )

evaluate all points in the task

send the results back to $S M_{i, j}$

if $\left(S M_{i, j}\right.$ is responding to a blocking request) then

loop := "nonblocking"

end if

case ("no point" from $S M_{i, j}$ )

if $\left(S M_{i, j}\right.$ is at status busy) then

set $S M_{i, j}$ 's status idle

$C_{\text {idle }}:=C_{\text {idle }}+1$

end if

exit INLOOP

case ("all done" from $S M_{i, j}$ )

if $\left(S M_{i, j}\right.$ is at status busy) then

set $S M_{i, j}$ 's status idle

$C_{\text {idle }}:=C_{\text {idle }}+1$

end if

remove $S M_{i, j}$ from the master list

$C_{\text {active }}:=C_{\text {active }}-1$

if $\left(C_{\text {active }}=0\right)$ then

cycle INLOOP (will wait for "terminate")

end if

exit INLOOP

case (a "non-blocking" request from a worker)

reply "all done" to this worker $\left(W_{i}\right.$ was a master $)$ 


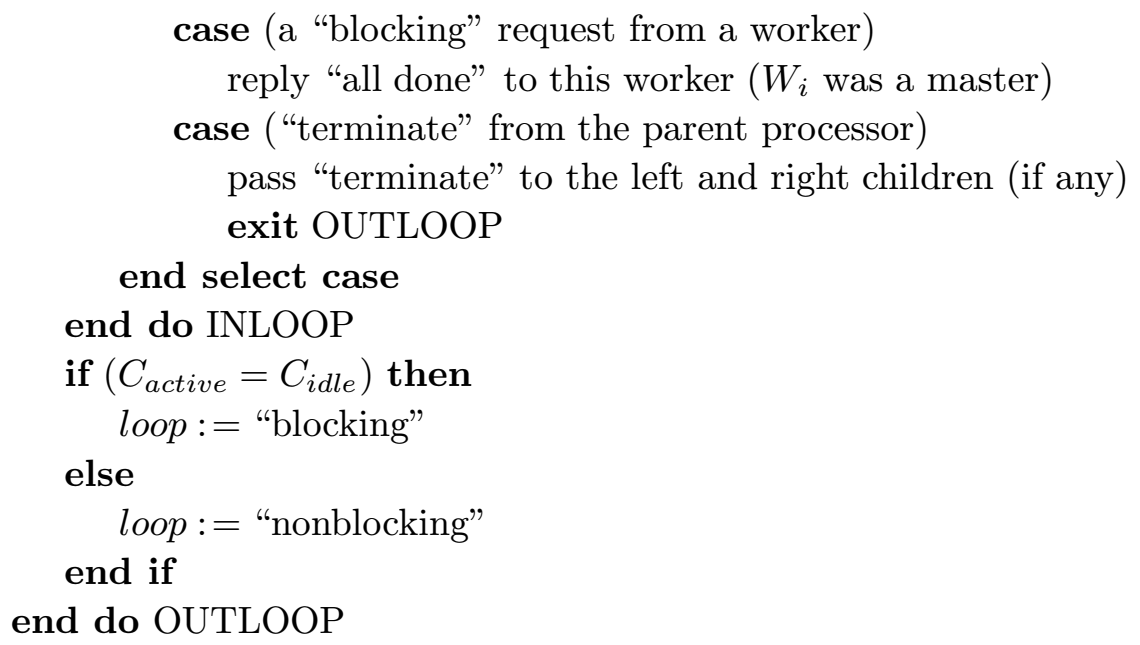

Pseudocode 3.3.

At the same time, the master $S M_{i, j}$ is generating sample points and responding to worker requests as described in the following Pseudocode 3.4.

$S_{i, j}$ : the portion of global convex hull boxes for $S M_{i, j}$

$Q_{w}$ : the blocked worker queue

$A_{b}(1: k):=0$ (the array of counters for tracking the number of blocking requests from workers $\left.W_{1}, W_{2}, \ldots, W_{k}\right)$

$C_{\text {new }}:=0$ (the counter for new points)

$C_{\text {send }}:=0$ (the counter for points that have been sent to workers)

$C_{\text {eval }}:=0$ (the counter for evaluated new points)

$N_{b i n}$ : the upper limit on the number of evaluation tasks sent to a worker at one time mesg: the message received from others

if $\left(S_{i, j}\right.$ is empty) then release all blocked workers in $Q_{w}$ (send them "no point" messages) else

find the longest dimensions for all boxes in $S_{i, j}$

$C_{\text {new }}:=$ the number of all newly sampled points along longest dimensions if $\left(Q_{w}\right.$ is not empty) then

loop sending at most $N_{\text {bin }}$ number of points to each worker in $Q_{w}$ with a tag

"responding to your blocking request"

update $C_{\text {send }}$

release the remaining blocked workers (if any)

\section{end if}

do while $\left(C_{\text {eval }}<C_{\text {new }}\right)$

keep waiting for any messages

select case (mesg)

case (a "non-blocking" request from $W_{i}$ )

if $\left(C_{\text {send }}<C_{\text {new }}\right)$ then

send at most $N_{b i n}$ number of points to $W_{i}$

update $C_{\text {send }}$

else 


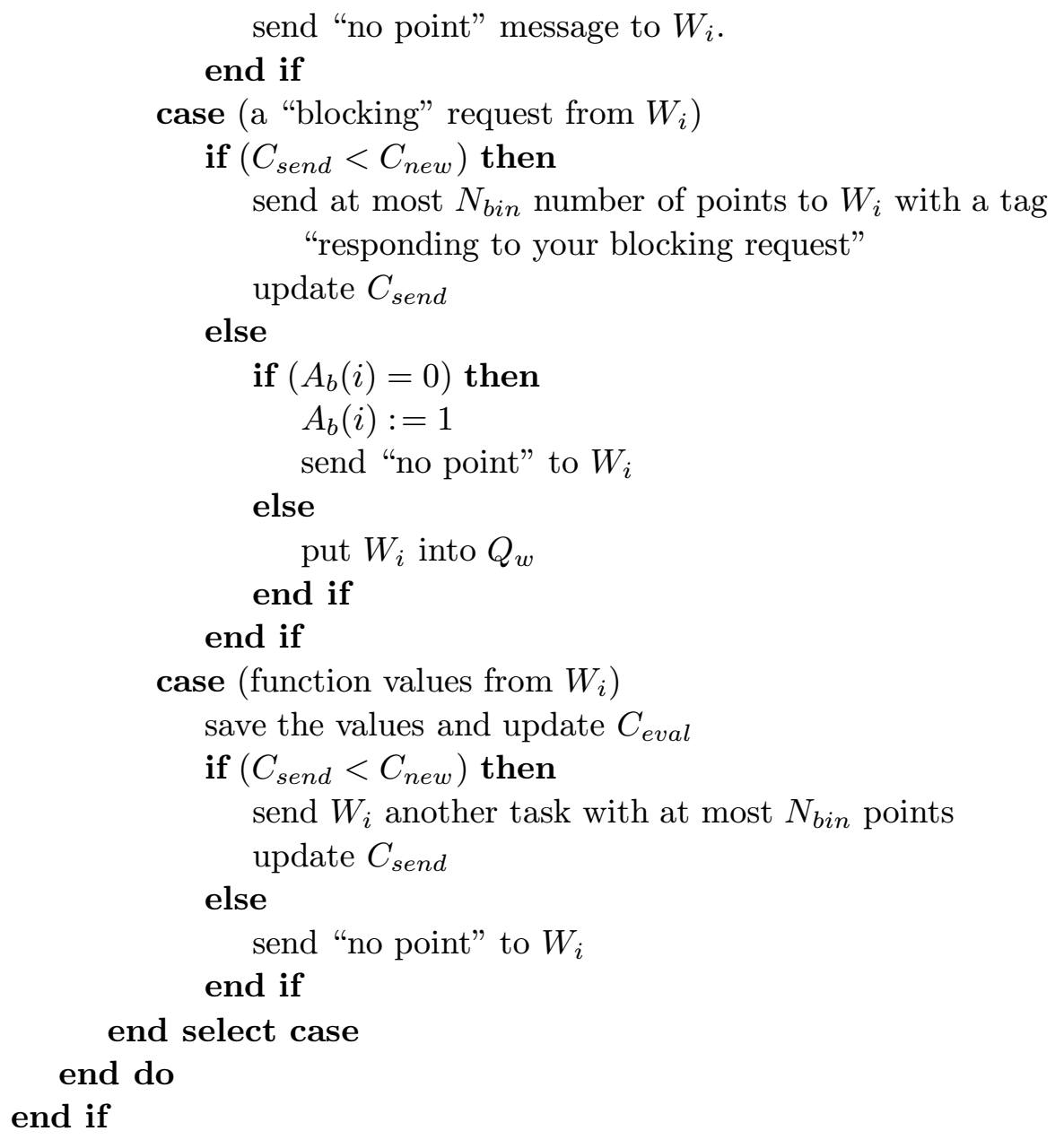

Pseudocode 3.4.

At the beginning of each iteration, $S M_{i, j}$ sends evaluation tasks to its blocked workers. If it has more blocked workers than tasks, it signals the remaining blocked workers to start a new cycle of requesting work from other masters. Otherwise, $S M_{i, j}$ keeps receiving function values from workers and sending out more tasks. When $S M_{i, j}$ is out of tasks, it notifies workers that are requesting tasks and queues up the workers that are blocked waiting for the next iteration. An array of blocking status $\left(A_{b}\right)$ is used to track the number of times that a worker has sent a blocking request to this master during this iteration. After the first blocking request from a worker, $S M_{i, j}$ tells the worker to continue seeking work from other masters. After the second blocking request from that same worker, during this iteration, $S M_{i, j}$ queues up that worker; this gives workers a better chance to find masters who have just become busy. Observe that the subdomain masters within the same subdomain need to synchronize with each other to find global convex hull boxes during every iteration; however, no synchronization or communication is needed among workers, and masters from different subdomains also work independently, until the final termination starts.

When all the masters are out of work at the end of an iteration, the next iteration begins, and the masters from different subdomains may start the next iteration at different times. Therefore, a master should encourage a worker, who has sent it the first blocking request, to seek work again from other masters. This asynchronous design allows a large number of workers to be used efficiently across masters and subdomains. Empirical results have shown that workers achieve a better load balance for a multiple subdomain search than for a single domain search. In comparison, workers 
Table 4.1. Test functions.

\begin{tabular}{ll}
\hline$\#$ & Description \\
\hline 1 & $f=x \cdot x / 3000$ \\
2 & $f=-\sqrt{\sum_{i=1}^{N} x_{i}-0.5(i-1)} / N$ \\
3 & $\left.f=1+\sum_{i=1}^{N} x_{i}{ }^{2} / 500-\prod_{i=1}^{N} \cos \left(x_{i} / \sqrt{i}\right)\right)$ \\
4 & $f=\sum_{i=1}^{N} 2.2 \times\left(x_{i}+0.3\right)^{2}-\left(x_{i}-0.3\right)^{4}$ \\
5 & $f=\sum_{i=1}^{N} \sum_{j=1}^{i} x_{j}{ }^{2}$ \\
6 & $f=\sum_{i=1}^{N} 100\left(x_{i+1}-x_{i}^{2}\right)^{2}+\left(1-x_{i}\right)^{2}$ \\
7 & $f=10 N+\sum_{i=1}^{N} x_{i}^{2}-10 \cos \left(2 \pi x_{i}\right)$ \\
8 & Budding yeast parameter estimator $[17]$ \\
\hline
\end{tabular}

in pDIRECT_I work only for a fixed master, so they have to sit idle when the master runs out of work until the next iteration starts.

This scheme also naturally distributes tasks to workers according to the speed at which they finish the work, unlike the load balancing methods that attempt to distribute an approximately equal number of function evaluation tasks to each worker. These methods assume that (1) the function evaluation at different coordinates costs the same computationally and/or (2) each worker finishes the function evaluation within the same amount of time. In fact, these two assumptions are not satisfied in many parallel systems, even though some are claimed to be homogeneous. Most importantly, many engineering design problems do have different computation cost for different regions. Therefore, the measure of a reasonable load balancing should not be the equal quantities of tasks that are distributed among workers, but the degree that all of the workers are kept busy. Note that this scheme adds a parameter $N_{\text {bin }}$ used for stacking function evaluations to one evaluation task. It reduces the communication overhead when the objective function is cheap. However, $N_{b i n}$ should be set to 1 if the objective function is expensive. Otherwise, fewer tasks are available to workers and a load imbalance occurs.

\section{Performance results}

This section presents performance results regarding the main design issues discussed in the last section. The test functions used are described in Table 4.1; the first seven have the same initial domain $[-2,3]^{N}$. For some experiments, dimension $N=150$ and an artificial time delay $T_{f}$ are used to make the first seven test functions comparable to the 143-parameter estimation problem from computational biology.

\subsection{Data structures}

The size of the data structures is the number $C_{\text {real }}$ of 64 -bit REAL variables in the box data structures. Table 4.2 compares $I_{\text {out }}$ (the number of iterations when the memory is used up) with and without LBC and computes the percentage of $C_{\text {real }}$ reduction in LBC for runs with $I_{\max }=I_{\text {out }}$ without LBC. All tests failed to allocate memory when $C_{\text {real }}$ reaches $\approx 150 \cdot 10^{6}$ on System X. LBC reduces the size of the data structures by $20-50 \%$ for all the test functions $1-7$ and by $37 \%$ for the budding yeast problem. As the number of box columns grows larger without limit, a single master runs fewer iterations without LBC than with LBC. 
Table 4.2. Comparison of experiments without (NON-LBC) and with LBC for Test Functions 1-7.

\begin{tabular}{|c|c|c|c|c|c|}
\hline \multirow[b]{2}{*}{$\#$} & \multicolumn{2}{|c|}{ NON-LBC } & \multicolumn{3}{|c|}{ LBC } \\
\hline & $\overline{I_{\text {out }}}$ & $C_{\text {real }} / 10^{6}$ & $C_{\text {real }} / 10^{6}$ & $\%$ diff. & $I_{\text {out }}$ \\
\hline 1 & 159 & 153 & 112 & 26 & 273 \\
\hline 2 & 79 & 164 & 77 & 52 & 647 \\
\hline 3 & 213 & 162 & 111 & 31 & 391 \\
\hline 4 & 90 & 163 & 82 & 50 & 1000 \\
\hline 5 & 286 & 164 & 124 & 24 & 467 \\
\hline 6 & 163 & 160 & 109 & 31 & 328 \\
\hline 7 & 78 & 162 & 85 & 47 & 377 \\
\hline
\end{tabular}

Table 4.3. Comparison of $N_{g c}, N_{l c}$, and $N_{d}$ at the last iteration $I_{\max }$.

\begin{tabular}{ccccc}
\hline$\#$ & $N_{g c}$ & $N_{l c}$ & $N_{d}$ & $\frac{\left(N_{d}-N_{l c}\right)}{N_{d}}$ \\
\hline 1 & 58 & 2605 & 33358 & $92 \%$ \\
2 & 89 & 1228 & 6805 & $81 \%$ \\
3 & 145 & 2056 & 22375 & $90 \%$ \\
4 & 3 & 3201 & 6756 & $52 \%$ \\
5 & 140 & 1830 & 20276 & $90 \%$ \\
6 & 144 & 1276 & 28003 & $95 \%$ \\
7 & 144 & 3531 & 20614 & $82 \%$ \\
8 & 20 & 159 & 6545 & $97 \%$ \\
\hline
\end{tabular}

\subsection{Task allocation}

The following experiments demonstrate the effectiveness of the SELECTION implementation, task partition, and worker assignment in pDIRECT_II.

\subsubsection{SELECTION efficiency}

Table 4.3 compares $N_{g c}$ (number of global convex hull boxes), $N_{l c}$ (the combined number of local convex hull boxes), and $N_{d}$ (the combined number of different box diameters) on 32 subdomain masters for all test functions at the last iteration $\left(I_{\max }=1000\right.$ for Test Function $1-7$ and $I_{\max }=100$ for the budding yeast problem). In pDIRECT $I, M M_{i, 1}$ collects $N_{d}$ boxes and finds the convex hull box set. In pDIRECT_II, $N_{l c}$ local convex hull boxes are found by $S M_{i, j}(j=1, \ldots, n)$, then gathered on $S M_{i, 1}$, which identifies the global convex hull boxes. This approach increases the concurrency of the SELECTION implementation. Table 4.3 shows that it reduces the amount of data transferred over the network and the buffer size by $50-90 \%$ for all test functions.

\subsubsection{Task partition}

The following experiment is to study the parallel performance of the horizontal and vertical 1-D partition schemes. A total of $P=288$ processors are used. In the horizontal partition, each run has all $P$ masters that evaluate objective functions locally. In the vertical partition, a single master sends evaluation tasks to $P-1$ workers, each task holding $N_{b i n}=1$ set of point coordinates. Table 4.4 shows the timing results for Test Function 6 with $N=150$, given two function evaluation costs: $T_{f}=0.1$ second (the artificial case) and the original cost $T_{f}=0.0$ (less than $1.0 \mathrm{E}-7$ second). 


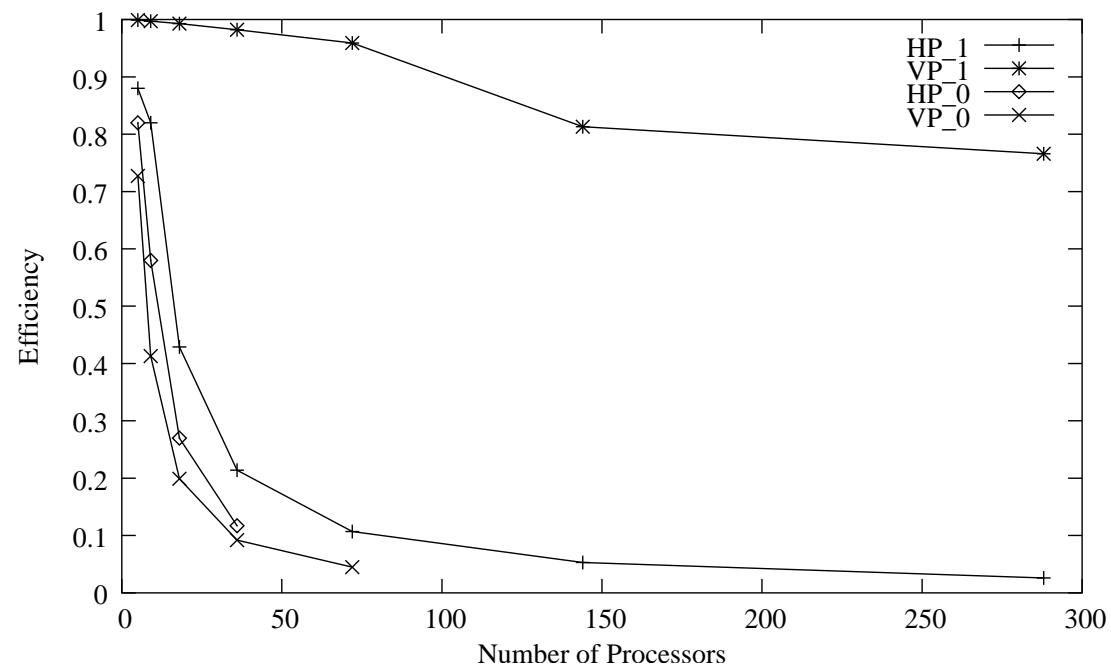

Figure 4.1. Comparison of the parallel efficiencies with different partition schemes and objective function costs.

Set $I_{\max }=300$ for the original cost and $I_{\max }=90$ for the artificial cost. To ensure that the two partition schemes are comparable in the number of processors, the timing results are measured from $P=3$ processors (if $P=1$, no workers are used in the vertical 1-D partition). Note that the horizontal 1-D partition has all $P$ processors available for function evaluations, while the vertical 1-D partition has only $P-1$ processors for that. Therefore, the parallel efficiency is estimated with the base $=3$ processors for the horizontal 1-D partition and base $=2$ for the vertical 1-D partition. The efficiency $E$ is thus computed as $\left(T_{b a s e} / T_{P}\right) /(P /$ base $)$ as shown in Figure 4.1.

Table 4.4. Parallel timing results (in seconds) for different function evaluation costs $T_{f}$ for Test Function 6 with $N=150$.

\begin{tabular}{|c|c|c|c|c|c|c|c|c|}
\hline \multicolumn{9}{|c|}{ Horizontal 1-D partition } \\
\hline$T_{f} \backslash \bar{P}$ & 3 & 5 & 9 & 18 & 36 & 72 & 144 & 288 \\
\hline 0.0 & 21.04 & 15.36 & $\overline{12.03}$ & 12.78 & 14.88 & 40.71 & 23.37 & 31.49 \\
\hline 0.16 & 6785.88 & 4614.23 & 2741.11 & 2633.46 & 2634.53 & 2636.03 & 2652.78 & 2644.96 \\
\hline \multicolumn{9}{|c|}{ Vertical 1-D partition } \\
\hline$T_{f} \backslash \bar{P}$ & $\bar{P}$ & 5 & 9 & 18 & 36 & 72 & 144 & 288 \\
\hline 0.0 & 49.93 & 41.18 & 40.28 & 41.81 & 45.10 & 45.53 & 65.10 & 55.07 \\
\hline 0.18 & 8720.27 & 4361.30 & 2184.76 & 1033.28 & 507.14 & 256.09 & 149.95 & 79.27 \\
\hline
\end{tabular}

When $T_{f}=0.0$ second, the horizontal 1-D partition (HP_0) runs faster than the vertical 1-D partition (VP_0). The masters in HP_0 locally evaluate the cheap objective function, thus avoiding the communication overhead for talking to workers. However, HP_0 does not achieve any speedup for $P=72,144$, and 288, because the number of convex hull boxes is insufficient to keep all masters busy (some masters have no convex hull boxes, thus evaluating no points locally). As for VP_0, the communication overhead always dominates the execution cost and it fails to achieve any speedup after $P$ reaches 144 . When $T_{f}=0.1$ second, the vertical 1-D partition (VP_1) runs more efficiently than the horizontal 1-D partition (HP_1) (see Figure 4.1) except for $P=3$. When $P=3$, three masters are evaluating functions for HP_1, while only two workers are doing so for VP_1. Nevertheless, all runs with $P \geq 5$ of VP_1 take a much shorter time than those of HP_1. The first 
Table 4.5. Comparison of theoretical parallel execution time $T_{t}$ and the parallel timing results $T_{p}$ with different hybrid partition schemes for Test Function 6 with $N=150, T_{f}=0.1$ sec, and $I_{\max }=90$. $P_{100}$ stands for using a total of 100 processors. $W_{100}, W_{200}$ stand for using a total of 100, 200 workers, respectively.

\begin{tabular}{rrrrrrrr}
\hline & \multicolumn{6}{c}{ Number of Masters } \\
\cline { 2 - 8 } schemes & & 1 & 2 & 4 & 8 & 16 & 32 \\
\hline$P_{100}$ & $T_{p}$ & 203.42 & 204.20 & 207.79 & 215.44 & 234.15 & 282.38 \\
& $T_{t}$ & 180.10 & 181.70 & 185.80 & 192.90 & 211.40 & 260.20 \\
& $T_{o}$ & 23.32 & 24.10 & 26.09 & 29.64 & 41.25 & 22.18 \\
& $E_{f}$ & $88.5 \%$ & $88.9 \%$ & $89.4 \%$ & $89.5 \%$ & $90.3 \%$ & $92.1 \%$ \\
\hline$W_{100}$ & $T_{p}$ & 201.53 & 185.41 & 184.83 & 184.88 & 185.61 & 187.29 \\
& $T_{t}$ & 178.00 & 178.00 & 178.00 & 178.00 & 178.00 & 178.00 \\
& $T_{o}$ & 23.53 & 7.41 & 6.83 & 6.88 & 7.61 & 9.29 \\
& $E_{f}$ & $88.3 \%$ & $96.0 \%$ & $96.3 \%$ & $96.3 \%$ & $95.9 \%$ & $95.0 \%$ \\
\hline$W_{200}$ & $T_{p}$ & 102.32 & 102.06 & 101.56 & 101.55 & 103.14 & 105.86 \\
& $T_{t}$ & 91.30 & 91.30 & 91.30 & 91.30 & 91.30 & 91.30 \\
& $T_{o}$ & 11.02 & 10.76 & 10.26 & 10.25 & 11.84 & 14.56 \\
& $E_{f}$ & $89.2 \%$ & $89.5 \%$ & $89.9 \%$ & $89.9 \%$ & $88.5 \%$ & $86.2 \%$ \\
\hline
\end{tabular}

reason is that the communication overhead is negligible compared to the objective function cost for VP_1. Secondly, when $P$ is large, no convex hull boxes are assigned to some masters, so they have to sit idle in the case of HP_1. In general, the number of convex hull boxes is much smaller than the number of function evaluations, because DIRECT samples two new points along each longest dimension for every convex hull box. Hence, the pure horizontal 1-D partition reaches its limits when $P$ is greater than the number of convex hull boxes. On the other hand, the memory limit on a single master makes the pure vertical 1-D partition impossible for runs with large $I_{\max }$. Therefore, a hybrid partition scheme is generally preferable to the pure partition schemes.

In the following two experiments, several hybrid partition schemes with different numbers of masters and workers are compared with the single master scheme for Test Function 6 with $N=150$ and $T_{f}=0.1$ second for a single subdomain. The first experiment varies the number of masters $\left(2^{i}, i=0, \ldots, 5\right)$ and fixes the total number of processors (implicitly, the number of workers also changes). The second experiment varies the number of masters and fixes the number of workers. $P=100$ (total number of processors) is used for Experiment 1. 100 and 200 workers, respectively, are involved in Experiment 2. The measured parallel execution timing results listed in Table 4.5 are compared to a theoretical lower bound defined by

$$
T_{t}=\sum_{i=1}^{I_{\max }}\left\lceil\frac{N_{i}}{k}\right\rceil T_{f},
$$

where $T_{t}$ is the theoretical lower bound on the parallel execution time, $N_{i}$ is the number of function evaluation tasks at iteration $i$, and $k$ is the total number of workers. $T_{t}$ depends on both the problem and the computing resource. It assumes all workers are fed continuously with evaluation tasks, distinct from reality, where finding convex hull boxes, synchronization, and communication all cost time as well. Clearly when $N_{i}$ is not exactly a multiple of $k$, some workers are idle during the last working cycle for that iteration. A worker ideally obtains either $\delta_{+}=\left\lceil N_{i} / k\right\rceil$ or $\delta_{-}=\left\lfloor N_{i} / k\right\rfloor$ number of tasks. The number $X$ of idle workers can be derived from

$$
\delta_{+}(k-X)+\delta_{-}(X)=N_{i}
$$




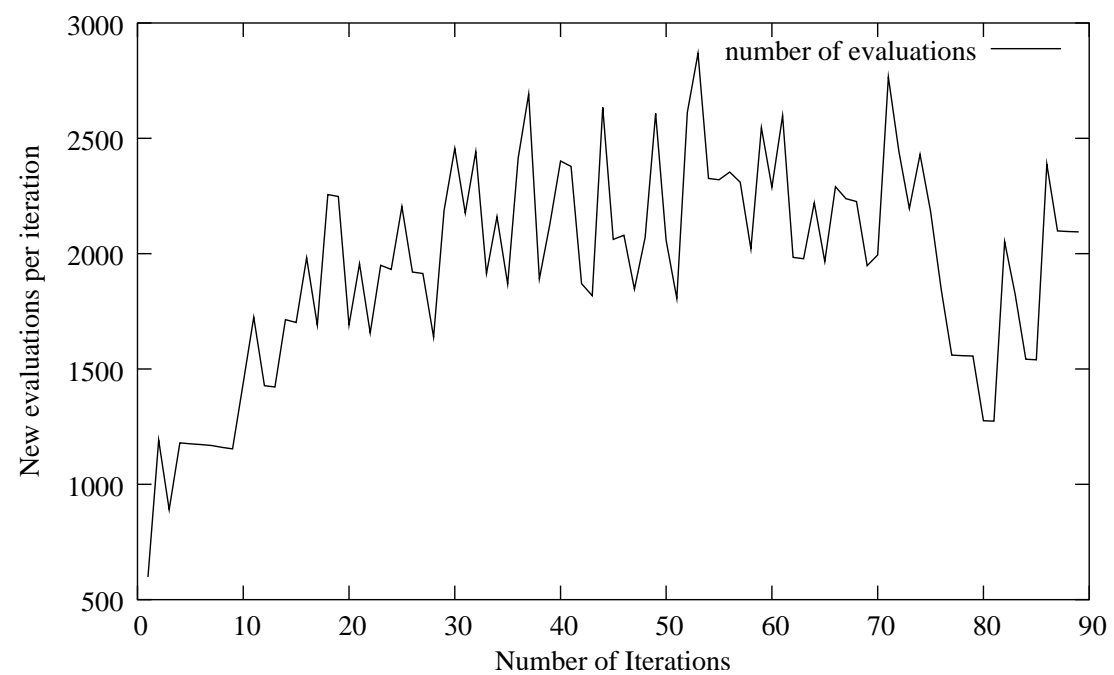

Figure 4.2. The plot of $N_{i}(i=1, \ldots, 90)$ for Test Function 6 with $N=150$.

Here, define the overhead of function evaluation $T_{o}=T_{p}-T_{t}$ and the efficiency of function evaluation $E_{f}=T_{t} / T_{p}$, where $T_{p}$ is the measured parallel execution time.

Figure 4.2 shows how the number of new evaluations per iteration $\left(N_{i}\right)$ changes for Test Function 6 with $N=150$ and $I_{\max }=90$. Given the number of workers, $T_{t}$ is then computed in Table 4.5, which shows that a wide range of hybrid task partition schemes perform reasonably well $\left(86.2 \% \leq E_{f} \leq 96.3 \%\right)$. In the case of $P_{100}, E_{f}$ grows slightly as the number of masters increases up to 32. Clearly, the number of convex hull boxes is sufficient to keep 32 masters busy. Moreover, smaller master-to-worker ratios seem to correspond to lower $E_{f}$ values. This phenomenon may indicate a potential bottleneck problem at masters that communicate with a great number of workers, or simply more idle workers. The above supposition was further investigated in the second experiment. In both $W_{100}$ and $W_{200}, E_{f}$ is improved at the beginning as the number of masters increases to a "peak point" with the best $E_{f}$. Then, it is degraded when the synchronization overhead among masters starts to dominate. Note that the peak point is 4 for $W_{100}$ and 8 for $W_{200}$, while the master-to-worker ratios at these two points are the same, 1:25. Moreover, $W_{200}$ has lower $E_{f}$ values than $W_{100}$ for the same number of masters, because masters in $W_{200}$ deal with more workers, and more workers in $W_{200}$ are likely to be idle. Also, the same amount of work distributed to 100 workers in $W_{100}$ generates more communication interactions between masters and workers in $W_{200}$. Therefore, the search with a single subdomain will always eventually decrease $E_{f}$ as more and more workers are used.

\subsubsection{Worker assignment}

The following experiment demonstrates that function evaluation tasks are allocated more efficiently for a multiple subdomain search than for a single subdomain search. Load balancing among workers is improved greatly with the globally shared worker pool of pDIRECT_II, especially when masters of different subdomains generate unequal amounts of work. $P=320$ processors were used for the budding yeast problem. $P=200$ processors were used for the artificial test problems (Test Functions 1-7) with $N=150$ and $T_{f}=0.1$ sec.

In this experiment, the feasible domain is split into four subdomains. Note that the same test problem with four split subdomains can be solved in three different ways using $P$ processors. 
Table 4.6. Comparison of $T_{a}, T_{b}$, and $T_{c}$ in seconds.

\begin{tabular}{rrrrrr}
\hline$\#$ & \multicolumn{1}{c}{$T_{a}$} & \multicolumn{1}{c}{$T_{b}$} & \multicolumn{1}{c}{$T_{c}$} & $\frac{\left(T_{b}-T_{a}\right)}{T_{a}}$ & $\frac{\left(T_{c}-T_{a}\right)}{T_{a}}$ \\
\hline 1 & 382 & 407 & 441 & $6.2 \%$ & $15.4 \%$ \\
2 & 1132 & 1139 & 1185 & $0.6 \%$ & $4.6 \%$ \\
3 & 358 & 369 & 417 & $3.1 \%$ & $16.4 \%$ \\
4 & 870 & 874 & 921 & $0.4 \%$ & $5.9 \%$ \\
5 & 260 & 263 & 312 & $1.1 \%$ & $20.0 \%$ \\
6 & 428 & 476 & 477 & $11.2 \%$ & $11.4 \%$ \\
7 & 1142 & 1148 & 1196 & $0.5 \%$ & $4.7 \%$ \\
8 & 8595 & 10643 & 11059 & $23.8 \%$ & $28.7 \%$ \\
\hline
\end{tabular}

Table 4.7. Comparison of total number of subdomain function evaluations for experiments listed in Table 4.6. $e_{i}$ is the total number of evaluations for subdomain $i$, which is the same for all of the ways (a), (b), and $(\mathrm{c})$.

\begin{tabular}{rrrrrrr}
\hline$\#$ & $e_{1}$ & $e_{2}$ & \multicolumn{1}{c}{$e_{3}$} & \multicolumn{1}{c}{$e_{4}$} & $\bar{e}$ & \multicolumn{1}{c}{$s^{2}$} \\
\hline 1 & 181409 & 194927 & 194927 & 181463 & 2090.9 & $7789.1^{2}$ \\
2 & 550691 & 550691 & 550691 & 550691 & 6118.8 & 0 \\
3 & 176075 & 176075 & 176075 & 176075 & 1956.4 & 0 \\
4 & 421723 & 421723 & 421723 & 421723 & 4685.8 & 0 \\
5 & 123685 & 123685 & 123685 & 123685 & 1374.3 & 0 \\
6 & 228193 & 203635 & 198727 & 192397 & 2286.0 & $15661^{2}$ \\
7 & 555435 & 555435 & 555435 & 555435 & 6171.5 & 0 \\
8 & 82471 & 44631 & 47531 & 87063 & 1635.6 & $22445^{2}$ \\
\hline
\end{tabular}

(a) All $P$ processors are used to run a multiple subdomain search with four subdomains. The parallel execution time is $T_{a}$.

(b) Four single subdomain searches are run in parallel, each using $P / 4$ processors on one of the four subdomains. The overall parallel execution time $T_{b}$ is the longest duration of all four runs.

(c) Four single subdomain searches are run sequentially, each using all $P$ processors on each of the four subdomains. The parallel execution time $T_{c}$ is the total duration of these four runs.

Table 4.6 compares $T_{a}, T_{b}$, and $T_{c}$ for all the test problems $\left(I_{\max }=90\right.$ for Test Functions $1-7$ and $I_{\max }=40$ for the budding yeast problem). $T_{a}$ is the smallest among the three. $T_{b}$ is only slightly bigger than $T_{a}$ for Test Functions $2,3,4,5$, and 7 , but becomes significantly bigger $(>5 \%)$ for Test Functions 1, 6, and 8, each of which has a large $s^{2}$, the variance of the total number of function evaluations for the four subdomains (in Table 4.7). Also, $T_{c}$ is the largest among the three. Observe that it is only slightly bigger $(<5 \%)$ than $T_{a}$ for Test Functions 2 and 7 . Table 4.7 shows that these two test functions have a large $\bar{e}=\sum e_{i} / I_{\max }(>6000)$, the average number of function evaluations per iteration, where $e_{i}$ is the total number of evaluations for subdomain $i$. Since more tasks are generated at each iteration for Test Functions 2 and 7 than for the other test functions, $P-1$ workers are better load balanced in case (c).

Figure 4.3 shows the normalized workload among workers for two runs: (1) Test Function 6 with moderate $\bar{e}$ and large $s^{2}$, and (2) Test Function 7 with large $\bar{e}$ and small $s^{2}$. Generally, workload is normalized by dividing the total evaluation time for each worker by the average evaluation time 
Table 4.8. Normalized workload ranges (WR) of a, b, and c for experiments listed in Table 4.6.

\begin{tabular}{lcccc}
\hline \multicolumn{1}{c}{$\#$} & $W R_{a}$ & $W R_{b 1}$ & $W R_{b 2}$ & $W R_{c}$ \\
\hline 1 & {$[0.99969,1.0002]$} & {$[0.95743,1.0413]$} & {$[0.99193,1.0091]$} & {$[0.97495,1.0250]$} \\
width & $5.4685 \mathrm{E}-5$ & $8.3845 \mathrm{E}-2$ & $1.7152 \mathrm{E}-2$ & $5.0023 \mathrm{E}-2$ \\
2 & {$[0.99985,1.0002]$} & {$[0.99871,1.0014]$} & {$[0.99871,1.0014]$} & {$[0.9959,1.0049]$} \\
width & $3.2897 \mathrm{E}-4$ & $2.6460 \mathrm{E}-3$ & $2.6460 \mathrm{E}-3$ & $9.7536 \mathrm{E}-3$ \\
3 & {$[0.99972,1.0001]$} & {$[0.99633,1.0038]$} & {$[0.99633,1.0038]$} & {$[0.98213,1.0138]$} \\
width & $3.8 \mathrm{E}-4$ & $7.4827 \mathrm{E}-3$ & $7.4827 \mathrm{E}-3$ & $3.1646 \mathrm{E}-2$ \\
4 & {$[0.99989,1.0001]$} & {$[0.99840,1.0017]$} & {$[0.99841,1.0017]$} & {$[0.99470,1.0032]$} \\
width & $2.1 \mathrm{E}-4$ & $3.2577 \mathrm{E}-3$ & $3.2576 \mathrm{E}-3$ & $8.4916 \mathrm{E}-3$ \\
5 & {$[0.99967,1.0005]$} & {$[0.99476,1.0062]$} & {$[0.99478,1.0063]$} & {$[0.98949,1.0104]$} \\
width & $7.9529 \mathrm{E}-4$ & $1.1487 \mathrm{E}-2$ & $1.1488 \mathrm{E}-2$ & $2.0914 \mathrm{E}-2$ \\
6 & {$[0.99973,1.0001]$} & {$[0.93170,1.1108]$} & {$[0.99497,1.0038]$} & {$[0.98802,1.0072]$} \\
width & $4.0281 \mathrm{E}-4$ & $1.7910 \mathrm{E}-1$ & $8.8312 \mathrm{E}-3$ & $9.9186 \mathrm{E}-1$ \\
7 & {$[0.99984,1.0001]$} & {$[0.99898,1.0011]$} & {$[0.99898,1.0011]$} & {$[0.99678,1.0035]$} \\
width & $2.3958 \mathrm{E}-4$ & $2.0986 \mathrm{E}-3$ & $2.0986 \mathrm{E}-3$ & $6.7307 \mathrm{E}-3$ \\
\hline
\end{tabular}

for all workers. Specially for case (b), the workload is also computed based on $P / 4$ processors for each run instead of considering all $P$ processors for the general normalization. Table 4.8 lists the normalized workload ranges, where $W R_{b 1}$ was obtained by averaging the workload based on $P$ processors and $W R_{b 2}$ was obtained by averaging the workload based on $P / 4$ processors.

Figure 4.3 plots the normalized workload among workers for Test Functions 6 and 7 in cases (a), (b), and (c). Figure 4.3(1)I and II use different ways of normalization for case (b). In all three pictures of Figure 4.3, case (a) has the best load balancing, i.e., the workload values fluctuate in the narrowest range around the average value 1.0 as listed in Table 4.8. In Figure 4.3(1)I, case (b) presents the widest range $\left(W R_{b 1}\right)$ and an interesting pattern that correlates with the variance of the number of function evaluations for the four subdomains of Test Function 6. In case (c), the workload values fall within ranges slightly wider than those in case (a). Nevertheless, if the average workload is computed separately (based on $P / 4$ processors) for each run of case (b), case (b)'s thus computed average workload range $\left(W R_{b 2}\right)$ for Test Function 6 is slightly narrower than that of case (c), but wider than that of case (a). This explains the timing results for Test Function $6\left(T_{a}<T_{b} \approx T_{c}\right)$. Since $s^{2}$ is 0 for Test Function 7 , the workload pattern is regular for all four runs of case (b) as shown in Figure 4.3(2). Also, the same workload range is obtained by either averaging the workload of all workers $\left(W R_{b 1}\right)$ or of the workers within each run $\left(W R_{b 2}\right)$ of case (b). Similarly for Test Function 7, the narrower workload range implies shorter parallel execution time. This experiment concludes that the multiple subdomain search has the best parallel performance in terms of parallel execution time and load balancing. Multiple subdomain search allows masters from different subdomains to provide work to the globally shared workers at different times, especially for subdomains that generate different amounts of work. In comparison, all masters run out of tasks at the end of each iteration in the single subdomain search. In the latter case, therefore, all workers will be idle until new tasks are available at the next iteration, a direct consequence of the DIRECT algorithm's data dependency. 


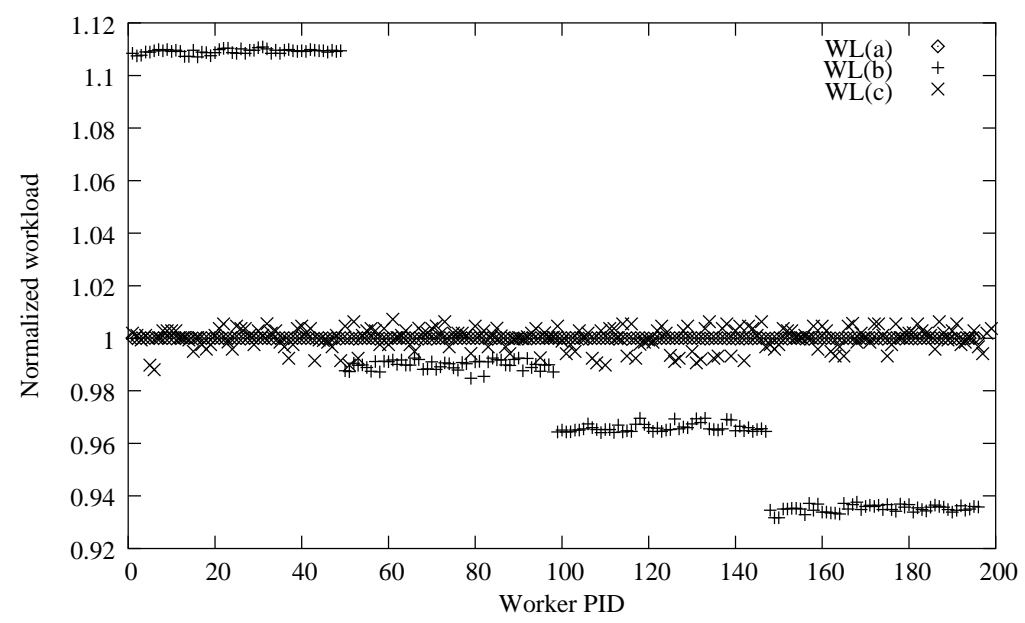

I. Normalization based on $P$ processors for case (b).

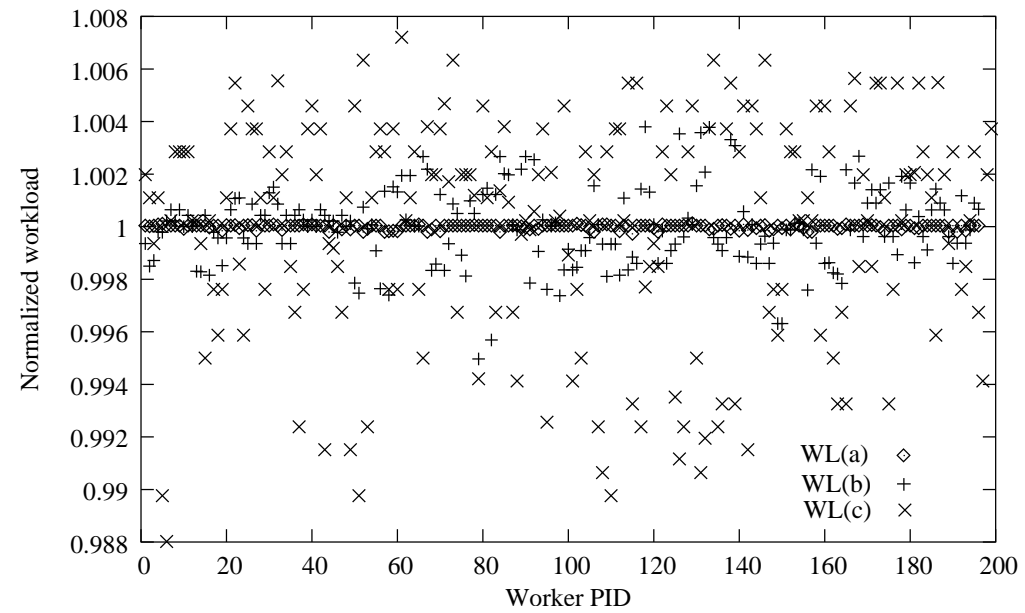

II. Normalization based on $P / 4$ processors for case (b).

(1) Test Function 6.

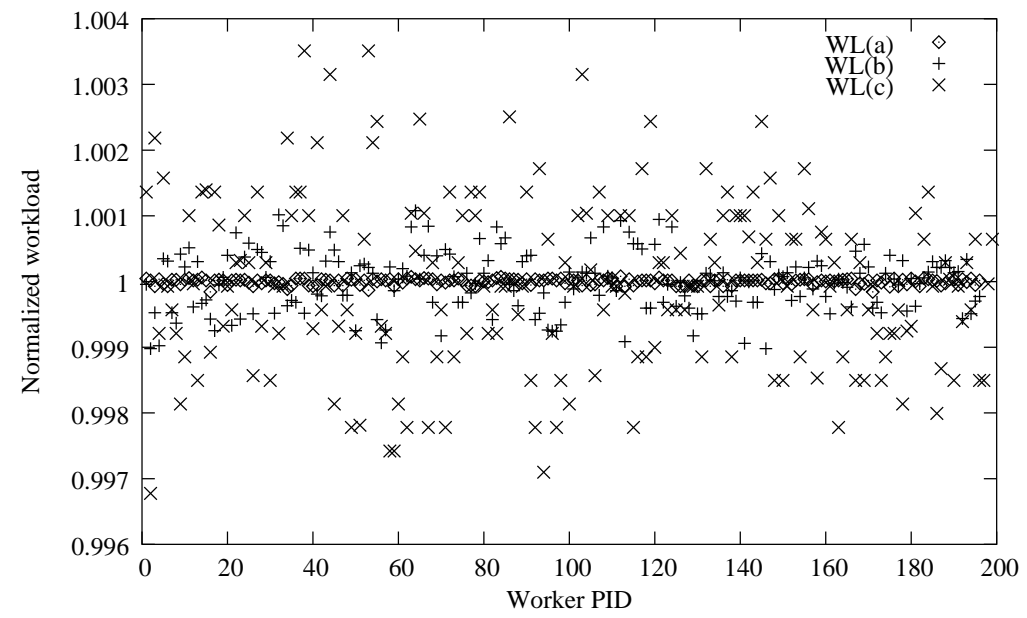

(2) Test Function 7.

Figure 4.3. Comparison of the workload (WL) patterns among workers for cases (a) (circles), (b) ("+" marks), and (c) ("x" marks). 


\section{Conclusions and future work}

This paper describes the pertinent considerations and rationale during the evolution of several massively parallel DIRECT implementations. Several memory reduction techniques and scalability improvements in the parallel scheme have been used in the largest application of DIRECT known to the authors - solving 143-dimensional optimization problems on up to 320 processors in parallel. Several design decisions were analyzed and supported by experiments. Future research in parallel DIRECT may consider the following venues. First, memory reduction techniques that take advantage of the limit on the number of iterations can be explored further. In particular, one may consider limits that take several box columns into account. Second, one may try to mitigate the effect of data dependency by guessing which points DIRECT will sample in the next iteration, and proactively sampling them when many workers are idle at the end of the current iteration. Intuitively, several points whose values are yet unknown should not substantially affect the convex hull, so one may be able to precompute certain objective function values ahead of time (effectively for free, by using otherwise idle workers), and replace expensive function evaluations with cheap table lookups later. Third, improving the implementation efficiency of convex hull computation and memory reduction techniques will likely allow parallel DIRECT to scale to even larger problems on even larger machines. This is especially important when the objective function is cheap, and thus the overhead of internal bookkeeping is significant.

\section{Acknowledgment}

This work was supported in part by AFRL Grant F30602-01-2-0572 and AFOSR Grant F4962002-1-0090.

\section{References}

1. M. D. Atkinson, J.-R. Sack, N. Santoro, and T. Strothotte, "Min-max heap and generalized priority queues", Communications of the ACM, vol. 29, no. 10, pp. 996-1000, 1986.

2. C. A. Baker, L. T. Watson, B. Grossman, R. T. Haftka, and W. H. Mason, "Parallel global aircraft configuration design space exploration", in High Performance Computing Symposium 2000, A. Tentner (Ed.), Soc. for Computer Simulation Internat, San Diego, CA, 2000, pp. 101-106.

3. M. C. Bartholomew-Biggs, S. C. Parkhurst, and S. P. Wilson, "Global optimization approaches to an aircraft routing problem", EUR J. Operational Research, vol. 146, no. 2, pp. 417-431, 2003.

4. W. R. Esposito and C. A. Floudas, "Global optimization in parameter estimation of nonlinear algebraic models via the Error-In-Variables approach", Ind Eng. Chemistry and Research, vol. 37, pp. 1841-1858, 1998.

5. J. M. Gablonsky, "Modifications of the DIRECT algorithm", PhD thesis, Department of Mathematics, North Carolina State University, Raleigh, NC, 2001.

6. C. Gau and M. A. Stadtherr, "Nonlinear parameter estimation using interval analysis", in AIchE Symposium, vol. 94, no. 320, pp 445-450, 1999.

7. A. Grama, A. Gupta, G. Karypis, and V. Kumar, Introduction to Parallel Computing, Pearson Education Limited, 2nd Edition, 2003.

8. W. Gropp, E. Lusk, and R. Thakur, Using MPI-2: Advanced features of the message-passing interface, The MIT Press, Cambridge, Massachusetts, London, England, 1999.

9. J. He, A. Verstak, L. T. Watson, T. S. Rappaport, C. R. Anderson, N. Ramakrishnan, C. A. Shaffer, W. H. Tranter, K. Bae, and J. Jiang, "Global optimization of transmitter placement in wireless communication systems", in Proc. High Performance Computing Symposium 2002, A. Tentner (ed.), Soc. for Modeling and Simulation International, San Diego, CA, pp. 328-333, 2002. 
10. J. He, M. Sosonkina, C. A. Shaffer, J. J. Tyson, L. T. Watson, and J. W. Zwolak, "A hierarchical parallel scheme for global parameter estimation in systems biology", in Proc. 18th Internat. Parallel \& Distributed Processing Symp., CD-ROM, IEEE Computer Soc., Los Alamitos, CA, 2004.

11. J. He, M. Sosonkina, L. T. Watson, A. Verstak, and J. W. Zwolak, "Data-distributed parallelism with dynamic task allocation for a global search algorithm", in Proc. High Performance Computing Symposium 2005 , M. Parashar and L. Watson (eds.), Soc. for Modeling and Simulation Internat., San Diego, CA, 2005, pp. $164-172$.

12. J. He, L. T. Watson, N. Ramakrishnan, C. A. Shaffer, A. Verstak, J. Jiang, K. Bae, and W. H. Tranter, "Dynamic data structures for a direct search algorithm", Computational Optimization and Applications, vol. 23, no. 1, pp. 5-25, 2002.

13. D. R. Jones, C. D. Perttunen, and B. E. Stuckman, "Lipschitzian optimization without the Lipschitz constant", J. Optim. Theory Appl., vol. 79, no. 1, pp. 157-181, 1993.

14. D. R. Jones, "The DIRECT global optimization algorithm", in Encyclopedia of Optimization, vol. 1, Kluwer Academic Publishers, Boston, 2001, pp. 431-440.

15. C. G. Moles, P. Mendes, and J. R. Banga, "Parameter estimation in biochemical pathways: a comparison of global optimization methods", Genome Res., vol. 13, pp. 2467-2474, 2003.

16. J. Nieplocha and B. Carpenter, "ARMCI: A portable remote memory copy library for distributed array libraries and compiler run-time systems", in 3rd Workshop on Runtime Systems for Parallel Programming (RTSPP) of International Parallel Processing Symposium, IPPS/SDP'99, CDROM, 1999.

17. T. D. Panning, L. T. Watson, N. A. Allen, K. C. Chen, C. A. Shaffer, and J. J. Tyson, "Deterministic global parameter estimation for a model of the budding yeast cell cycle", J. Global Optim., to appear.

18. K. Parzyszek, J. Nieplocha, and R. A. Kendall, "A generalized portable SHMEM library for high performance computing", in 12th IASTED International Conference Parallel and Distributed Computing and Systems (PDCS), pp. 401-406, 2000.

19. L. T. Watson and C. A. Baker, "A fully-distributed parallel global search algorithm", Engineering Computations, vol. 18, no. 1/2, pp. 155-169, 2001.

20. J. Zhou, X. Deng, and P. Dymond, "A 2-D parallel convex hull algorithm with optimal communication phases", Parallel Computing, vol. 27, no. 3, pp. 243-255, 2001. 